\title{
Composition, Distribution and Characterization of Polybrominated Diphenyl Ethers (PBDEs) in the Soil in Taiyuan, China
}

\author{
K. Li $\cdot$ S. Fu $\cdot$ Z. Z. Yang $\cdot$ X. B. Xu
}

Received: 20 June 2008/Accepted: 2 October 2008/Published online: 15 October 2008

(C) Springer Science+Business Media, LLC 2008

\begin{abstract}
A total of 21 surface soil samples covering the whole territory of Taiyuan city were collected for analysis the 39 polybrominated diphenyl ethers (PBDEs). The total PBDEs concentration ranged from 0.016 to $211.416 \mathrm{ng} \mathrm{g}^{-1}$ in urban soils and 5.961 to $144.162 \mathrm{ng} \mathrm{g}^{-1}$ in industrial plant soils. PBDE homologues analysis and principal component analysis (PCA) revealed that the major source of PBDEs in Taiyuan might be potentially associated with the prevalent use of deca-BDE as a flame retardant. Furthermore, increasing PBDEs contamination was observed in north Taiyuan because of the degree of urbanization and the distribution of industrial plants.
\end{abstract}

Keywords China Contamination $\cdot$ Polybrominated diphenyl ethers · Soil

Polybrominated diphenyl ethers (PBDEs) are man-made chemicals widely used as flame retardants in a wide variety of plastics, textiles and electronic components (Pirard and Pauw 2007). Technical PBDEs are synthesized by electrophilic substitution of diphenyl ethers. Therefore $2,2^{\prime}, 4,4^{\prime}$-PBDE isomers dominate in the three commercial formulations: penta-, octa- and deca-brominated diphenyl

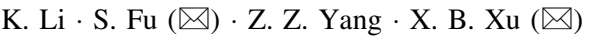

State Key Laboratory of Environmental Chemistry and Ecotoxicology, Research Center for Eco-Environmental Sciences, Chinese Academy of Sciences, P.O. Box 2871, Beijing 100085, People's Republic of China

e-mail: fu_shan@sina.com

X. B. Xu

e-mail: xuxb@rcees.ac.cn
}

ethers, named after their mean bromine content (de Wit 2002). PBDEs have the potential for endocrine disruption, bioaccumulation, and long-range transport (Moon et al. 2007a), while an increasing number of studies have been conducted with regard to their carcinogenic and mutagenic properties (Knoth et al. 2007; Naert et al. 2007). Recently, certain commercial mixtures of PBDEs (penta and octa formulations) were banned in Europe because of their persistence and potential environmental and human health risks (Kemmlein et al. 2003). However, the demand for PBDEs has been increasing rapidly, and in Asia all commercial BDE mixtures are used without regulation (Tan et al. 2007).

Soil is an important reservoir for many persistent organic pollutants (POPs) including PBDEs. Hence, as POPs are released or escape into the environment, the burden in soils globally is a complex function of the balance between inputs and losses. A full understanding of the fate of PBDEs requires knowledge of their concentrations and distributions in the soil of all major ecological zones. However, there is a shortage of data from China. More information about the contamination status, environmental behavior and source-sink relationships of PBDEs in soil is therefore needed to be better understood.

The city of Taiyuan is located in central Shanxi province, China. The city occupies an area of $6,956 \mathrm{~km}^{2}$ with $180 \mathrm{~km}^{2}$ classified as urban area. Urban area mainly centralized in the eastern part of Taiyuan with 3 million urban residents. The climate is dominated by temperate semi-wet monsoon, with a mean annual temperature of $9.6^{\circ} \mathrm{C}$. China clay is the main representative soil type in Taiyuan. With the development of industry in recent years, Shanxi province has provided increasing amounts of chemical products and secondary energy, such as coke and electric power. At the same time, environmental contamination has become 
more and more serious, but the levels of PBDE residues in the soil of Taiyuan have not been investigated in detail.

Because of the paucity of data on levels of PBDEs in soil in China, we undertook this study to investigate the composition, distribution and characterization of PBDEs in soil samples obtained from urban areas of Taiyuan. The main objectives of the present study were to identify the possible sources of pollution, and to explore possible factors affecting contamination.

\section{Materials and Methods}

Surface soil samples $(0-10 \mathrm{~cm}$ depth) were obtained from 15 urban sites and 6 industrial plants in Taiyuan, Shanxi Province (Fig. 1), in January 2006. The industrial plants site 16-21 were thermoelectric plant, fertilizer plant, chemical plant, building site, coking plant and No. 1 power plant, respectively. Any overlying vegetation was removed before samples of the surface soil were collected in triplicate using a hand-held coring device. Each composite urban soil sample consisted of 16 sub-samples in the same grid pattern at each sampling site. Each composite industrial plant soil sample consisted of 3 sub-samples. The gathered samples were freeze-dried, mixed thoroughly, sieved to 60 mesh (International standard size, $250 \mu \mathrm{m}$ ), transferred to amber glass and stored at $4^{\circ} \mathrm{C}$. The remaining water content in the soil was determined gravimetrically after drying individual composite soil samples in air at $105^{\circ} \mathrm{C}$ for $12 \mathrm{~h}$. All results are reported on a dry weight basis.

A standard solution of 39 PBDE congeners (EPA method 1614 standard solution) (Accustandard, New Haven, CT, USA) was used to quantify the following mono-through hepta-brominated BDEs: mono-BDEs 1, 2, and 3; di-BDEs 7, 8, 10,11, 12, 13, and 15; tri-BDEs 17, $25,28,30,32,33,35$, and 37; tetra-BDEs $47,49,66,71$, 75 , and 77; penta-BDEs $85,99,100,116,118,119$, and 126; hexa-BDEs 138, 153, 154, 155, and 166; and heptaBDEs 181, 183, and 190. A standard solution of PBDE congeners (EO-5278) (Cambridge Isotope Laboratories, USA) was purchased from Cambridge Isotope Laboratories, which was used for quantitation of BDE-205, 206, 209. Standard reference material (SRM) NIST-2585 (reference house dust for organic contaminants analysis) was obtained from NIST, USA. All solvents used were pesticide grade (J. T. Baker, USA).

Five grams of each urban surface soil sample or three grams of each industrial plant soil sample were weighed then ground with anhydrous sodium sulfate into a freeflowing powder. The samples were extracted with $40 \mathrm{~mL}$ of hexane/dichloromethane $(1: 1, \mathrm{v} / \mathrm{v})$ by ultrasonication for 4 min then separated by centrifugation. This process was

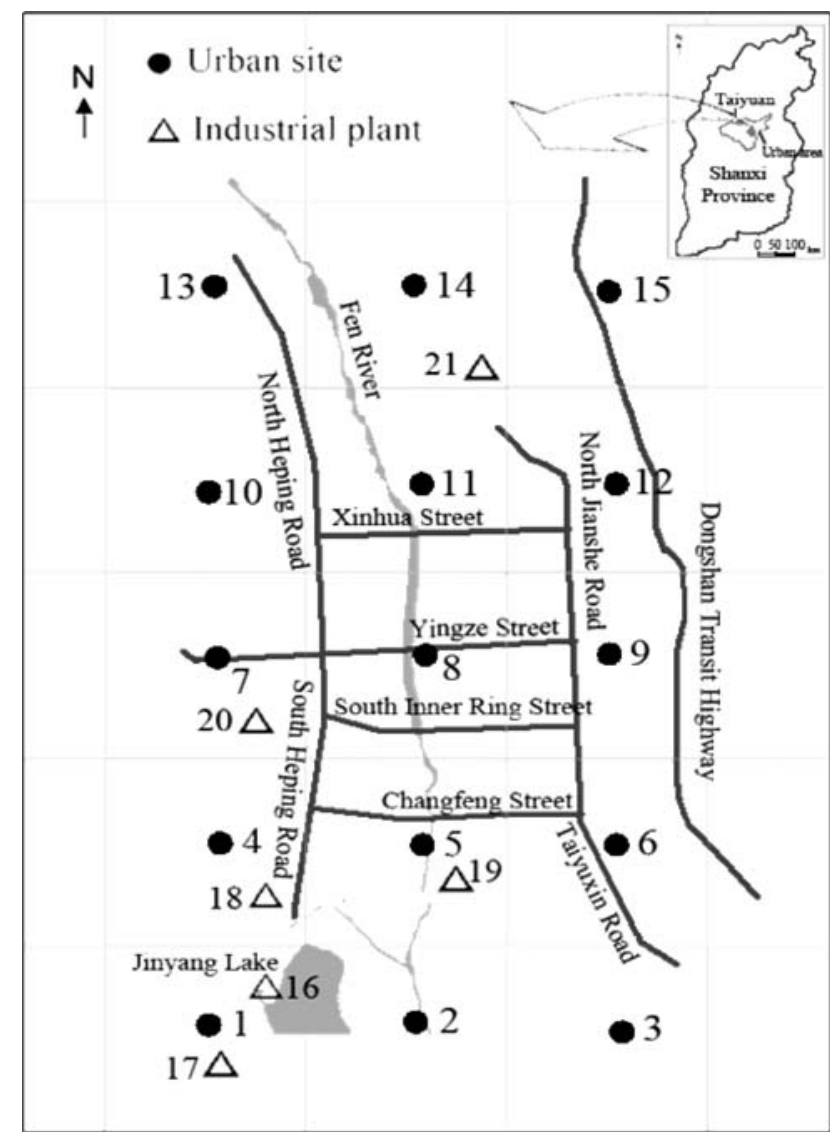

Fig. 1 Surface soil sampling sites in urban areas of Taiyuan

repeated three times. The concentrated extracts were then cleaned on a 15-mm i.d. column packed from the bottom to top with $1 \mathrm{~g}$ activated silica gel, $3 \mathrm{~g}$ basic silica gel, $1 \mathrm{~g}$ activated silica gel, $4 \mathrm{~g}$ acid silica gel (44\% concentrated sulfuric acid, w/w), $4 \mathrm{~g}$ acid silica gel (22\% concentrated sulfuric acid, w/w), $1 \mathrm{~g}$ activated silica gel and $1 \mathrm{~cm}$ anhydrous sodium sulfate. The first fraction was eluted with $100 \mathrm{~mL}$ hexane. The elution was evaporated with a rotary evaporator and then reduced to $2 \mathrm{~mL}$ under a gentle $\mathrm{N}_{2}$ stream. The concentrated extracts were then cleaned on a 10-mm i.d. column packed from the bottom to top with $2 \mathrm{~g}$ activated silica gel, $2 \mathrm{~g}$ silver nitrate silica $(10 \%, \mathrm{w} / \mathrm{w})$ and $1 \mathrm{~cm}$ anhydrous sodium sulfate. The first fraction was eluted with $40 \mathrm{~mL}$ hexane then the PBDEs were eluted with $40 \mathrm{~mL}$ of hexane/dichloromethane $(1: 1, \mathrm{v} / \mathrm{v})$. The elution was evaporated with a rotary evaporator and then reduced to $20 \mu \mathrm{L}$ under a gentle $\mathrm{N}_{2}$ stream for analysis. Throughout the extraction, cleanup and analysis procedure, the analytes were protected from light by wrapping the containers with aluminum foil or by using amber glassware.

We analyzed PBDEs with an Agilent 6890 series gas chromatograph (GC) coupled with an Agilent 5973 mass spectrometer (MS) using negative chemical ionization 
source in SIM mode. In negative chemical ionization mode, the MS source temperature was $150^{\circ} \mathrm{C}$, and the electron energy was $70 \mathrm{eV}$. The $\mathrm{GC}$ was equipped with a split-splitless injector held constant at $270^{\circ} \mathrm{C}$. Gas chromatographic separation was performed on a $15-\mathrm{m}$ DB-5MS capillary column $(30 \mathrm{~m} \times 0.25 \mathrm{~mm}$, internal diameter; $0.25-\mu \mathrm{m}$ film thickness). We maintained the GC column at $80^{\circ} \mathrm{C}$ for $1 \mathrm{~min}$ and then ramped at $10^{\circ} \mathrm{C} / \mathrm{min}$ to $200^{\circ} \mathrm{C}$, further ramped at $20^{\circ} \mathrm{C} / \mathrm{min}$ to $300^{\circ} \mathrm{C}$, and held at this temperature for $5 \mathrm{~min}$. The compounds were monitored at $\mathrm{m} / \mathrm{z} 79$ and 81 (for PBDE congeners), and m/z 486.7 and 488.7 (for BDE209 only).

A laboratory method control group was run to demonstrate the lack of interference and cross-contamination. In addition, a procedural blank was run in parallel for every set of 6 samples to further check for interference and crosscontamination. Duplicate samples were analyzed in the laboratory along with the regular samples as an additional quality control tool to ensure valid results. Instrument stability and relative response factor variance were determined by analyzing the calibration standard solutions in each sample batch.

Quantification of PBDE was performed using an external standard method. Three quality control criteria were used to ensure correct identification of the target compounds: (1) GC retention times matched those of the standard compounds within $\pm 0.05 \mathrm{~min}$; (2) the signal-tonoise ratio was greater than $3: 1$; (3) each compound had two monitored ions. Isotopic ratios between quantification and confirmation ions were within $\pm 15 \%$ of theoretical values. The SRM sample (NIST-2585) was analyzed to validate the analytical method employed. The results were satisfactory, with a z-score of $\leq 1$ for all congeners (range: $0.32-0.86$ for the PBDEs, $p<0.05$ ). The limits of detection (LOD) for PBDE were within the range of $0.001-$ $0.05 \mathrm{ng} \mathrm{g}^{-1}$ (dry weight) for di- to nona-BDE, and $0.1 \mathrm{ng} \mathrm{g}^{-1}$ (dry weight) for BDE209.

\section{Results and Discussion}

39 PBDEs were identified in all of the urban and industrial plant soil samples. The detection rates of PCBs in the soils were up to $100 \%$, which indicated wide occurrence of these compounds in Taiyuan city. The details were shown in Table 1. The concentrations of $\Sigma$ PBDE in the urban surface soil samples ranged from 0.016 to $211.416 \mathrm{ng} \mathrm{g}^{-1}$ (median $2.096 \mathrm{ng} \mathrm{g}^{-1}$, dry weight). The highest concentration was found at site 11 , followed site $9\left(52.951 \mathrm{ng} \mathrm{g}^{-1}\right)$. The lowest concentration was found at site 2 . The concentrations of $\Sigma$ PBDE in the industrial plant soils ranged from 5.961 to $144.162 \mathrm{ng} \mathrm{g}^{-1}$ (dry weight). The medians concentration in

Table 1 Total concentration of PBDEs in each sample

\begin{tabular}{|c|c|c|c|c|c|c|c|c|c|c|}
\hline Site & Di-BDEs & Tri-BDEs & Tetra- BDEs & Penta-BDEs & Hexa-BDEs & Hepta-BDEs & Octa-BDEs & Nona-BDEs & BDE-209 & $\sum$ PBDEs \\
\hline 1 & N.D. & 0.001 & 0.008 & 0.001 & 0.001 & 0.001 & N.D. & 0.002 & 0.008 & 0.022 \\
\hline 2 & N.D. & 0.001 & 0.007 & 0.001 & 0.001 & N.D. & N.D. & 0.001 & 0.006 & 0.016 \\
\hline 3 & N.D. & 0.001 & 0.007 & 0.001 & 0.001 & 0.001 & N.D. & 0.231 & 20.658 & 20.899 \\
\hline 4 & N.D. & 0.019 & 0.012 & 0.009 & 0.005 & 0.005 & N.D. & 0.022 & 0.216 & 0.286 \\
\hline 5 & 0.001 & 0.002 & 0.006 & 0.003 & 0.001 & 0.001 & N.D. & 0.008 & 0.079 & 0.100 \\
\hline 6 & 0.004 & 0.002 & 0.007 & 0.005 & 0.001 & 0.001 & N.D. & 0.046 & 1.196 & 1.216 \\
\hline 7 & N.D. & 0.003 & 0.015 & 0.017 & 0.009 & 0.018 & N.D. & 0.049 & 0.482 & 0.594 \\
\hline 8 & 0.006 & 0.009 & 0.028 & 0.072 & 0.084 & 0.026 & N.D. & 0.098 & 4.470 & 4.794 \\
\hline 9 & 0.004 & 0.003 & 0.008 & 0.006 & 0.002 & 0.004 & N.D. & 0.296 & 52.628 & 52.951 \\
\hline 10 & N.D. & 0.002 & 0.006 & 0.009 & 0.002 & 0.001 & N.D. & 0.176 & 1.901 & 2.096 \\
\hline 11 & N.D. & 0.023 & 0.055 & 0.056 & 0.027 & 0.044 & N.D. & 1.666 & 209.546 & 211.416 \\
\hline 12 & N.D. & 0.010 & 0.029 & 0.015 & 0.011 & 0.007 & N.D. & 0.447 & 26.268 & 26.788 \\
\hline 13 & N.D. & 0.005 & 0.006 & 0.006 & 0.003 & 0.002 & N.D. & 0.083 & 48.997 & 49.102 \\
\hline 14 & N.D. & 0.006 & 0.029 & 0.014 & 0.006 & 0.012 & N.D. & 0.337 & 15.298 & 15.701 \\
\hline 15 & N.D. & 0.003 & 0.010 & 0.003 & 0.002 & 0.004 & N.D. & 0.029 & 0.221 & 0.272 \\
\hline 16 & 0.005 & 0.109 & 0.472 & 0.173 & 0.073 & 0.136 & N.D. & 1.269 & 50.702 & 52.938 \\
\hline 17 & 0.043 & 0.057 & 0.074 & 0.060 & 0.028 & 0.062 & N.D. & 0.473 & 5.164 & 5.961 \\
\hline 18 & N.D. & 0.125 & 0.122 & 0.134 & 0.065 & 0.079 & N.D. & 0.773 & 16.797 & 18.094 \\
\hline 19 & N.D. & 0.208 & 0.139 & 0.427 & 0.158 & 0.347 & 0.017 & 1.480 & 34.601 & 37.377 \\
\hline 20 & N.D. & 0.129 & 0.042 & 0.028 & 0.019 & 0.175 & N.D. & 0.950 & 13.347 & 14.692 \\
\hline 21 & 0.040 & 0.156 & 0.195 & 0.142 & 0.062 & 0.912 & N.D. & 9.657 & 132.998 & 144.162 \\
\hline
\end{tabular}

Data units are in nanograms per gram; N.D. not detected 
Fig. 2 Total concentration of the PBDE homologues in each sample

\begin{tabular}{|c|c|c|c|c|}
\hline Q di-BDEs & 目 tri-BDEs & $\square$ tetra-BDEs & $\otimes$ penta-BDEs & : hexa-BDEs \\
\hline 血 hepta-BDEs & [:] octa-BDEs & $\mathbb{Z}$ nona-BDEs & BDE209 & \\
\hline
\end{tabular}

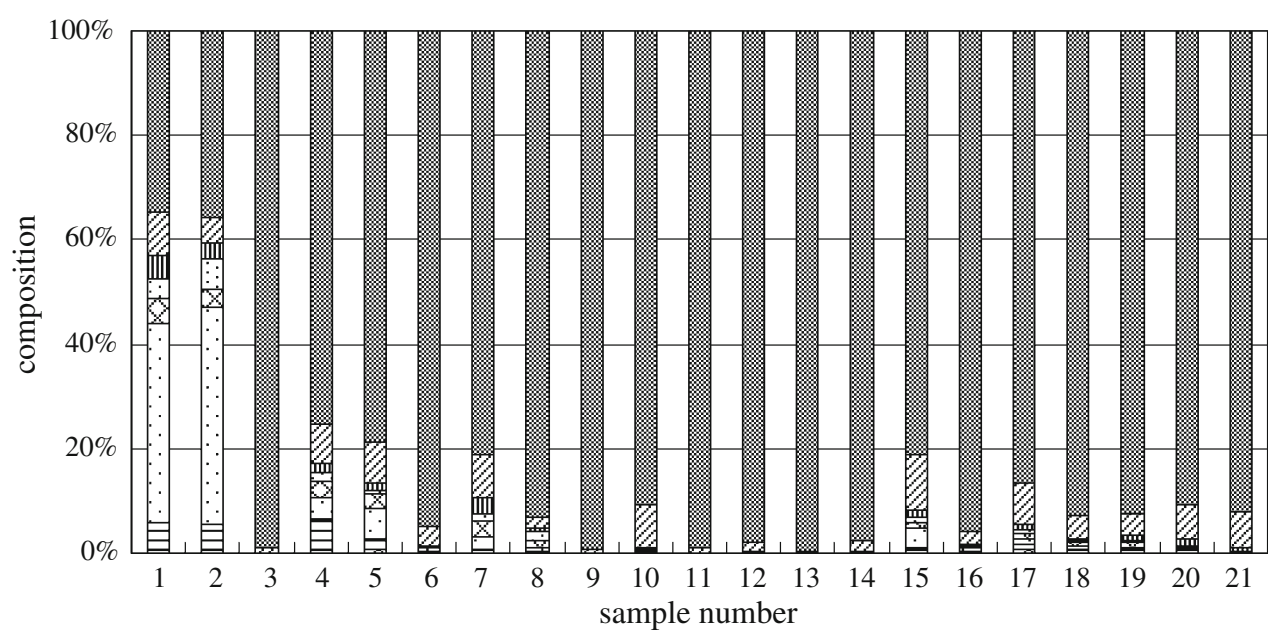

the soil of industrial plant was higher than those obtained in the soil of urban area.

We further compared the soil concentrations of PBDEs reported elsewhere in literature. In comparison with other areas of China, the levels were lower than those of the surface soil of e-waste recycling site Guiyu, Guangdong province (0.26-824 ng g ${ }^{-1}$ ) (Wang et al. 2005), and Pearl River Delta (0.028-70.5 $\mathrm{ng} \mathrm{g}^{-1}$ ) (Zou et al. 2007). In contrast to other countries, the levels were lower than those in background soils in the United Kingdom and Norway (65-12,000 $\mathrm{ng} \mathrm{g}^{-1}$ ) (Hassanin et al. 2004), and Izmir, Turkey (0.05-2840 ng g ${ }^{-1}$ ) (Cetin and Odabasi 2007). The contamination of soils in the present study had relatively slight PBDE contamination levels except for BDE209 on average.

Of the PBDE homologues, the dominant PBDE detected in this study was BDE209 accounting for more than $70 \%$ of the total PBDEs in most sampling locations (Fig. 2). BDE209 was also the main PBDE homologue in an e-waste pollution area in southeast China (Zou et al. 2007). Lower brominated congeners were detected at relatively low concentrations. The PBDE congener profile of the soil in Taiyuan was similar to that of technical deca-BDE products (Saytex 102E and Bromkal 82-0DE) (LaGuardia et al. 2006). This profile was reasonable since BDE209 firmly bound to particles due to its low volatile property.

Principal component analysis (PCA) was performed to evaluate similarities or differences between the PBDE congener patterns of each sample, all data were normalized to a percent of the sum of PBDEs. PCs were determined by eigenvalues of over 1 . Two extracted PCs could explain $75.7 \%$ of the data variance. A loading plot and score plot
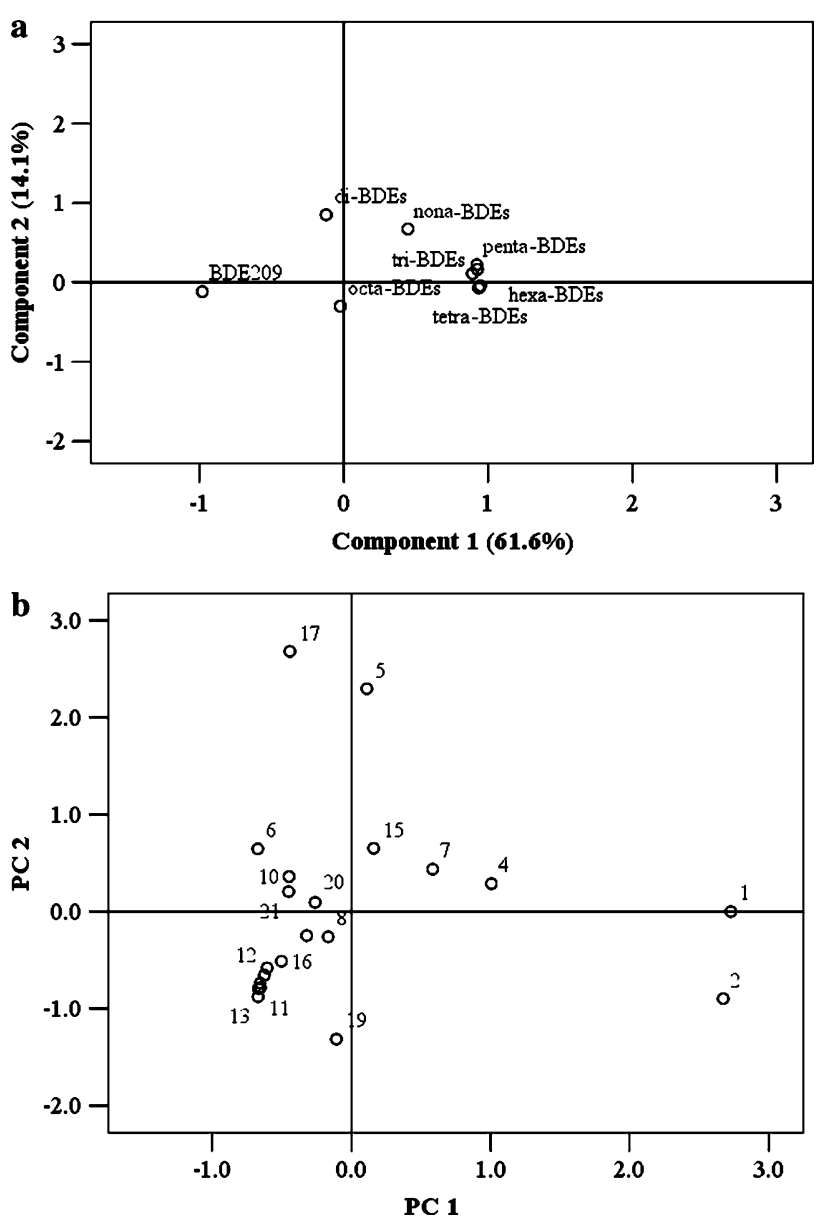

Fig. 3 a Two-dimensional principal component loading plot obtained from the data correlation matrix of 21 soil samples in Taiyuan. b Two-dimensional principal component score plot obtained from the data correlation matrix of 21 soil samples in Taiyuan 


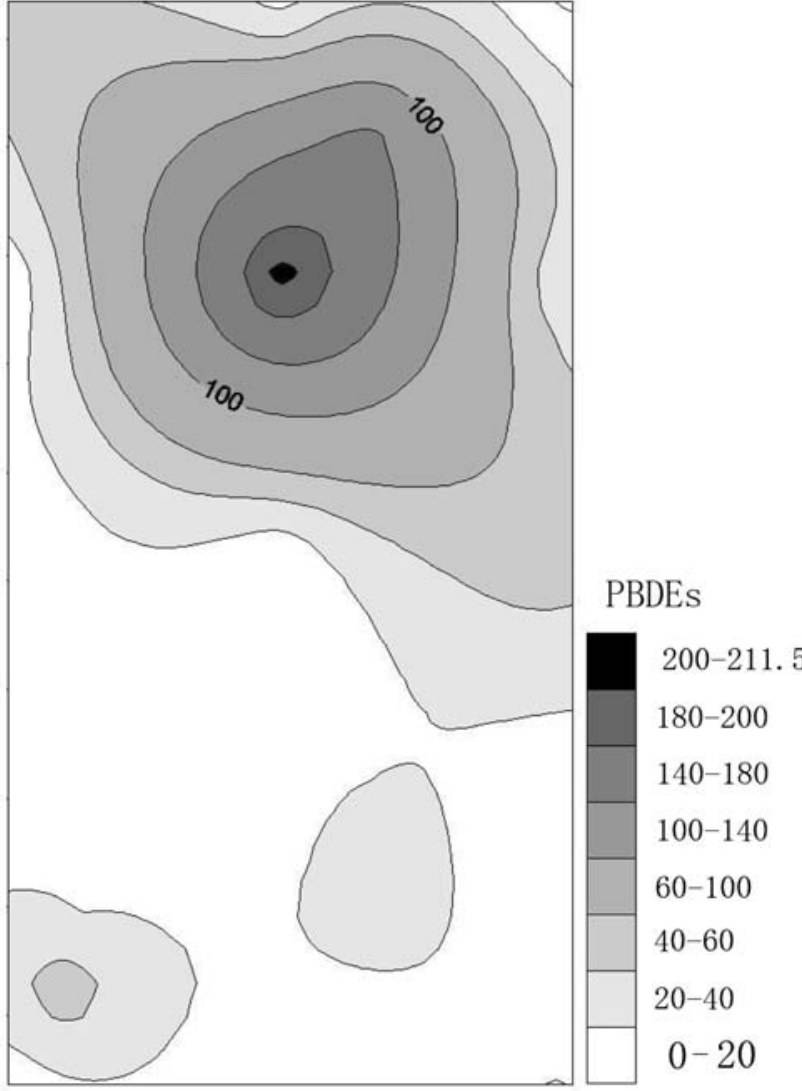

Fig. 4 The distributions of PBDEs in the soils in Taiyuan (data in the Figure are in units of $\mathrm{ng} \mathrm{g}^{-1}$ )

rotation were obtained using the varimax method (Fig. 3). The PCA results indicate that the compositions of PBDEs in the most Taiyuan samples were similar, possibly originating from the same source, and the mainly congener was BDE209. It could be concluded that the major source of PBDEs in Taiyuan may be potentially associated with the prevalent use of deca-BDE as a flame retardant.

It is interesting to explore a correlation between $\Sigma$ PBDE and total organic carbon content (TOC) in the soil samples. The 21 samples were heated at $450^{\circ} \mathrm{C}$ for $4 \mathrm{~h}$ in order to determine the total organic carbon content (Ball 1964). TOC content of the urban surface soil samples and the industrial plant soil samples ranged from $0.78-2.84 \%$ and $1.65-9.74 \%$, respectively. No significant correlation existed between the $\Sigma$ PBDE and total organic carbon content. These findings are consistent with the findings of a previous study conducted in sediment from Korean coast (Moon et al. 2007b).

The distribution of $\Sigma$ PBDEs in the soil samples shows in Fig. 4 revealing an increasing trend from south to north Taiyuan. Possible reason was the distribution of urban area that was mainly centralized in the north of Taiyuan. On the other hand, the south of Taiyuan was mainly suburb. The contamination status of urban area soils about PBDEs was higher than other suburb area of Taiyuan. It suggested that PBDEs were strongly associated with the degree of urbanization (Stapleton et al. 2005). Another possible reason, there was a correlation between the distributions of industrial plants and the contamination status in Taiyuan. The highest concentration was found at site 11, followed site 9. Some industrial plants (such as power plant) were mainly built in these areas. The contamination status was lower from these areas to surrounding areas gradually. And in south, there were two relatively higher areas where also built some industrial plants. The contamination of industrial plant soils was possibly a primary sink and emission source for PBDEs. Such reasons could possibly explain the contamination status of Taiyuan.

Acknowledgments This study was supported by National Natural Scientific Foundation of China (No. 20707031). We thank the research staff in Inst. Geochem. Geophys. Exploration. especially Prof. Cheng and Prof. Liu for sample collection and technical assistance.

\section{References}

Ball DF (1964) Loss-on-ignition as an estimate of organic soil matter and organic carbon in non-careous. J Soil Sci 15:84-92. doi: 10.1111/j.1365-2389.1964.tb00247.x

Cetin B, Odabasi M (2007) Particle-phase dry deposition and air-soil gas-exchange of polybrominated diphenyl ethers (PBDEs) in Izmir, Turkey. Environ Sci Technol 41:4986-4992. doi:10.1021/ es070187v

de Wit CA (2002) An overview of brominated flame retardants in the environment. Chemosphere 46:583-624. doi:10.1016/S00456535(01)00225-9

Hassanin A, Breivik K, Meijer SN, Steinnes E, Thomas GO, Jones KC (2004) PBDEs in European background soils: levels and factors controlling their distribution. Environ Sci Technol 38:738-745. doi:10.1021/es035008y

Kemmlein S, Herzke D, Law RJ (2003) BFR-governmental testing programme. Environ Int 29:781-792. doi:10.1016/S0160-4120 (03)00112-0

Knoth W, Mann W, Meyer R, Nebhuth J (2007) Polybrominated diphenyl ether in sewage sludge in Germany. Chemosphere 67:1831-1837. doi:10.1016/j.chemosphere.2006.05.113

LaGuardia MJ, Hale RC, Harvey E (2006) Detailed polybrominated diphenyl ether (PBDE) congener composition of the widely used penta-, octa-, and deca-PBDE technical flame-retardant mixtures. Environ Sci Technol 40:6247-6254. doi:10.1021/es060630m

Moon H-B, Kannan K, Lee S-J, Choi M (2007a) Atmospheric deposition of polybrominated diphenyl ethers (PBDEs) in coastal areas in Korea. Chemosphere 66:585-593. doi:10.1016/j.chemosphere. 2006.09.042

Moon H-B, Kannan K, Lee S-J, Choi M (2007b) Polybrominated diphenyl ethers (PBDEs) in sediment and bivalves from Korean coastal waters. Chemosphere 66:243-251. doi: 10.1016/j.chemosphere.2006.05.025

Naert C, Van Peteghem C, Kupper J, Jenni L, Naegeli H (2007) Distribution of polychlorinated biphenyls and polybrominated diphenyl ethers in birds of prey from Switzerland. Chemosphere 68:977-987. doi:10.1016/j.chemosphere.2007.01.009 
Pirard C, Pauw ED (2007) Absorption, disposition and excretion of polybrominated diphenyl ethers (PBDEs) in chicken. Chemosphere 66:320-325. doi:10.1016/j.chemosphere.2006.04.086

Stapleton HM, Dodder NG, Offenberg JH, Schantz MM, Wise SA (2005) Polybrominated diphenyl ethers in house dust and clothes dryer lint. Environ Sci Technol 39:925-931. doi:10.1021/ es0486824

Tan J, Cheng SM, Loganath A, Chong YS, Obbard JP (2007) Polybrominated diphenyl ethers in house dust in Singapore. Chemosphere 66:985-992. doi:10.1016/j.chemosphere.2006. 07.052
Wang D, Cai Z, Jiang G, Leung A, Wong MH, Wong WK (2005) Determination of polybrominated diphenyl ethers in soil and sediment from an electronic waste recycling facility. Chemosphere 60:810-816. doi:10.1016/j.chemosphere.2005.04.025

Zou M-Y, Ran Y, Gong J, Mai B-X, Zeng Ey (2007) Polybrominated diphenyl ethers in watershed soils of the Pearl River Delta, China: occurrence, inventory, and fate. Environ Sci Technol 41:8262-8267. doi:10.1021/es071956d 